\title{
Form Selection of Concomitant Polymorphs: A Case Study Informed by Crystallization Kinetics Modeling
}

\author{
Weiwei Tang${ }^{1}$, Yufeng Quan², Junbo Gong², Jingkang Wang², Qiuxiang Yin², and Tonglei \\ $\mathrm{Li}^{3}$
}

${ }^{1}$ Affiliation not available

${ }^{2}$ Tianjin University

${ }^{3}$ Purdue University System

May 5, 2020

\begin{abstract}
Molecular mechanisms and process kinetics of crystallizing concomitant polymorphs remain poorly understood. Solventmediated phase transformation is often mistaken as concomitant crystallization, mainly due to the two processes sharing similar kinetic profiles. Herein, we developed a population balance model to simulate a concomitant crystallization process of two polymorphs of tolfenamic acid (TFA). The kinetic modeling aims to better understand concomitant crystallization and help guide form selection of such a molecular system. Crystallization kinetics of ethanolic TFA solutions were uncovered from induction time measurements, as well as seeded and unseeded crystallization experiments. Both experimental and simulation results demonstrate that the stable form I crystallizes concomitantly with the metastable form II. The faster growing form II results in an intermediate decline in the kinetic profile of form I composition in crystallized samples, a characteristic feature of the concomitantly crystallized system. A four-quadrant scheme of attainable polymorph outcome was simulated under various crystallization conditions.
\end{abstract}

\section{Hosted file}

manucript-AICHEJ-Jan20_2020-V6-shorten.docx available at https://authorea.com/users/299606/

articles/429218-form-selection-of-concomitant-polymorphs-a-case-study-informed-by-

crystallization-kinetics-modeling 